\title{
Avaliação do desempenho cognitivo em idosos*
}

\author{
Evaluation of cognitive performance in the elderly
}

Pollyanna da Silva de Souza ${ }^{0}$ Jorge Tarcísio da Rocha Falcãob

Márcia Carréra Campos Leal ${ }^{c}$ Jacira Guiro Marinod

\section{Resumo}

Objetivo: avaliar o processo do envelhecimento, relacionando-o ao desempenho cognitivo. Método: estudo epidemiológico, descritivo, transversal, realizado na UNATI/UFPE, com 65 idosos. Foi utilizado questionário semiestruturado, incluindo dados sociodemográficos, escala de avaliação da satisfação global com a vida e o Miniexame do Estado Mental (MEEM). Resultados: 83,1\% apresentam uma doença ou condição crônica; 59,3\% - hipertensão, 18,5\% - problemas de coluna e artrose, 14,8\% - osteoporose, $13 \%$ - colesterol elevado, $11,1 \%$ - doença do coração, $9,3 \%$ - artrite e 3,7\% - diabetes. Foi reportada por $16,9 \%$ dos sujeitos a não-realização de atividades rotineiras por problema de saúde nas últimas duas semanas. Com relação à percepção acerca da própria saúde, $93,8 \%$ consideraram boa ou muito boa. No entanto, $66,2 \%$ mencionaram ter alguma dificuldade de memória, sendo esta freqüente para 58,1\% dos sujeitos com queixa, e rara ou muito rara para $41,9 \%$. Além disso, $73,8 \%$ praticam alguma atividade física: caminhada $(44,6 \%)$, a hidroginástica $(23,1 \%)$ e ioga $(13,8 \%)$. As análises inferenciais estatísticas evidenciaram efeito isolado significativo da escolaridade ( $F=8,703, p<0,000,64 \mathrm{~g} .1$.$) , mas nenhum efeito$ significativo isolado da idade $(\mathrm{F}=0,442, \mathrm{p}=0,724,64 \mathrm{~g} .1$. $)$ sobre os escores do MEEM. Conclusões: o processo de envelhecimento não deve ser associado necessariamente ao adoecimento e degeneração patológica, pois não inviabiliza o funcionamento sócio-psicocultural.

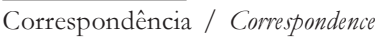

Pollyanna da Silva de Souza

Universidade Federal de Pernambuco - Av. Prof. Moraes Rego s/n, Cidade Universitária, 50739-970 - Recife-PE - Brasil - E-mail: pollysouza@ig.com.br

* Baseado em dissertação intitulada "Efeitos do envelhecimento sobre o desempenho cognitivo", apresentada em 19 de agosto de 2005 na pós-graduação em Psicologia Cognitiva da Universidade Federal de Pernambuco - UNATI/UFPE

\author{
Palavras-chave: \\ envelhecimento; \\ terapia cognitiva; \\ transtornos \\ cognitivos; estudos \\ epidemiológicos.
}




\section{Abstract}

Objective: this research aims to analyze the process of human ageing, concerning cognitive aspects. Method: epidemiologic-descriptive and transversal method was used. A sample of 65 elderly was constituted. Participants of this sample were interviewed about socio-demographic aspects and level of satisfaction with their lives, and were submitted to the Mini-Examination of Mental Condition (MEEM) Test. Results: $83.1 \%$ of participants reported at least one disease; $59.3 \%$ - hypertension, $18.5 \%$ - spine problems and arthritis, $14.8 \%$ osteoporosis, $13 \%$ - high cholesterol levels, $11.1 \%$ - heart diseases, $9.3 \%$ arthritis and $3.7 \%$ - diabetes. Among the participants, $16.9 \%$ reported interruption of daily activities due to a disease or organic malfunction, and a majority reported a good health condition. Nevertheless, $66.2 \%$ reported some degree of memory impairment, this problem being frequent for $58.1 \%$ of them. And $73.8 \%$ reported participation in some kind of physical activity: walking (44.6\%), hydrogymnastics (23.1\%) and yoga (13.8\%). Analysis of effects of variables showed isolated significant effect of school level over MEEM score $(\mathrm{F}=8.703, \mathrm{p}<0.000,64 \mathrm{~g} .1$. $)$, but no significant effect of age $(\mathrm{F}=0.442, \mathrm{p}=0.724$, 64g.1.). Conclusions: data collected here show that seniority cannot be necessarily associated to cognitive losses; ageing is not only an organic, biological process, but also a familiar, economic and cultural one.

Key words: aging; cognitive therapy; cognition disorders; epidemiologic studies.

\section{INTRODUÇÃO}

O desenvolvimento cognitivo na vida adulta e na velhice vem sendo um tema explorado na psicologia mundial nos últimos anos, visto que o envelhecimento populacional se configura como realidade demográfica em todo o mundo e não apenas no Brasil. Este teve, segundo $\operatorname{Veras}^{9}$ (2003), entre os anos de 1950 e 2002, um aumento da população idosa de $700 \%$, passando dos 2 milhões para 15,4 milhões em apenas 50 anos.

Esses estudos discutem principalmente dois aspectos relevantes no campo do desenvolvimento cognitivo em adultos: o primeiro diz respeito às perdas biológicas naturais do envelhecimento. É inegável que, com o pas- sar dos anos, as células, órgãos e sistemas do corpo humano comecem a apresentar déficits, havendo perdas em seu bom funcionamento.

O segundo se refere à cultura, que cada vez mais vem propiciando um envelhecimento saudável. Nesse sentido, teorias psicológicas vêm ressaltando as compensações culturais sobre eventuais perdas, privilegiando, por exemplo, investigações a respeito do desenvolvimento da sabedoria, demonstrando que pessoas idosas se beneficiam dessa característica ao serem comparadas com jovens no desempenho de tarefas cognitivas, o que será discutido pelos estudos relatados em seguida.

Um dos autores que desenvolvem pesquisas sobre esse tema, com o enfoque no envelhecimento cognitivo, é Paul B. Baltes, diretor 
do Lifespan Psychology, do Max Planck Institut for Human Development, em Berlim, Alemanha. Entre outras, duas linhas de pesquisa são desenvolvidas no instituto na área de psicologia cognitiva: envelhecimento cognitivo e psicologia da sabedoria.

Baltes e seus colaboradores investigam o que o autor chama de mecânica cognitiva e pragmática cognitiva (Baltes ${ }^{3}$, 1994). Lançando mão de uma metáfora computacional, propõe que a primeira (a mecânica cognitiva) é concebida como o hardware da inteligência, referindo-se à arquitetura neurofisiológica do cérebro, que é resultado da evolução da espécie. Em termos operacionais, a mecânica cognitiva diz respeito à velocidade e à precisão dos processos elementares de processamento do input da informação sensorial, memória motora e visual, além de processos de discriminação, comparação e categorização. Já a segunda, a pragmática cognitiva, diria respeito ao software da mente, ao conteúdo e sua funcionalidade em contextos culturais reais. A pragmática cognitiva tem relação com o tipo de conhecimento e de informação provenientes da cultura, como todos os conhecimentos a respeito do mundo e das ações humanas, que são adquiridos pelo processo de socialização do indivíduo. Incluem-se aqui a linguagem (leitura e escrita), qualificações educacionais, habilidades profissionais, conhecimento do self e habilidades que possibilitam lidar com situações da vida cotidiana.

Outras pesquisas evidenciaram que o desempenho de idosos é prejudicado quando se trabalha com reaprendizagem, revisão ou lembrança de informações recentes. Abando- nar ou recontextualizar conhecimentos prévios para que uma nova aprendizagem se dê parece mais difícil para idosos do que para adultos jovens; em outras palavras, esquecer parece ser mais difícil para os primeiros do que para os segundos (Kliegl; Linderberger, 1993, apud Baltes 3 , 1994). À medida que o grau de dificuldade e complexidade nas tarefas cognitivas aumenta, as diferenças entre desempenhos de idosos e jovens também aumentam, segundo Mayer e Kliegl (no prelo, apud Baltes $\left.{ }^{3}, 1994\right)$. Em tarefas mais fáceis, os idosos levam cerca de 50\% de tempo a mais que os adultos jovens, e quando as tarefas se tornam mais complexas, esse tempo passa a ser o dobro. Todas essas investigações sugerem que o déficit decorrente do envelhecimento afeta consideravelmente a mecânica cognitiva (hardware) e o funcionamento dos processos básicos de memória.

Observamos que estudos na área da psicologia do envelhecimento cognitivo apresentam algumas linhas de pesquisas consolidadas, como é o caso do grupo liderado por P. Baltes, em Berlim. Não obstante, estudos acerca do desenvolvimento cognitivo na fase adulta e na velhice são proporcionalmente menos freqüentes que aqueles direcionados ao desenvolvimento infantil. Sendo assim, este trabalho pretende contribuir com dados acerca da relação entre processo do envelhecimento e desempenho cognitivo.

\section{MÉTODO}

Realizou-se estudo epidemiológico, descritivo, transversal, do qual participaram 65 in- 
divíduos com idades a partir de 60 anos. Todos os idosos entrevistados eram brasileiros, residentes na região metropolitana de Recife (estado de Pernambuco). Esses sujeitos foram inicialmente entrevistados com base em questionário semi-estruturado para a caracterização da amostra em termos de dados sociodemográficos, ao que se seguiram a aplicação de escala de avaliação da satisfação global com a vida e o Miniexame do Estado Mental (MEEM), abaixo detalhados.

A amostra foi constituída por idosos alunos da Universidade Aberta à Terceira Idade (UnATI) da Universidade Federal de Pernambuco (UFPE), que foram convidados a participar da pesquisa. A UnATI foi criada em 1996, vinculada ao Programa do Idoso (PROIDOSO) da Pró-reitoria de Extensão da UFPE, e tem como finalidade a promoção de ações para melhoria da qualidade de vida das pessoas idosas, mediante atualização de seus conhecimentos e interação na sociedade contemporânea. A amostra foi calculada em 10\% do universo existente: 650 alunos matriculados no primeiro semestre de 2005 , em 29 cursos, no período da manhã e tarde, em diversas áreas, como por exemplo, Ioga, Línguas Estrangeiras (Espanhol, Italiano e Inglês), Educação e Saúde, Nutrição e Envelhecimento, dentre outros. Assim, a amostra foi constituída por 65 sujeitos, de ambos os sexos. Os participantes foram obtidos através de um sorteio aleatório realizado a partir da listagem de matrícula.

As pessoas convidadas a participar da pesquisa foram devidamente esclarecidas quanto às finalidades da mesma, e solicitadas a regis- trar sua livre aceitação de participação por escrito, em documento específico para tal fim (termo de consentimento livre e esclarecido), em conformidade com a Resolução no 196/ 96, do Conselho Nacional de Saúde, que regulamenta a investigação científica com seres humanos. Ainda em conformidade com a resolução acima, o projeto referente a esta pesquisa foi submetido e aprovado pelo Comitê de Ética em Pesquisa do Centro de Ciências da Saúde da Universidade Federal de Pernambuco (CEP/CCS/UFPE), protocolo de pesquisa $\mathrm{n}^{\circ} 054 / 2005$.

Os instrumentos utilizados para a realização do estudo foram os seguintes: a) um questionário semi-estruturado para caracterização da amostra, no qual foram solicitados dados de identificação pessoal, acrescido de b) uma escala de avaliação da Satisfação Global com a Vida (Neri ${ }^{8}$ 2001a apud Miranda ${ }^{7}$ 2005); c) o Min-Exame do Estado Mental (MEEM), usado para avaliar o desempenho cognitivo dos participantes (Antunes ${ }^{2}$ et al., 2005).

O questionário foi desenvolvido pela própria pesquisadora, com base na Pesquisa $\mathrm{Na}$ cional por Amostra de Domicílio (PNAD) do IBGE ${ }^{5}$ e perguntas pertinentes ao objetivo do estudo. A escala de Satisfação Global com a Vida é uma versão adaptada de Cantrill (1969 apud Miranda $\left.{ }^{7}, 2005\right)$, por $\operatorname{Neri}^{8}$ (2001a apud Miranda $\left.^{7}, 2005\right)$, que tem por objetivo uma auto-avaliação de estados subjetivos dos sujeitos.

O Miniexame do Estado Mental (MEEM) foi escolhido a partir de estudos realizados por Bertollucci ${ }^{4}$ et al. (1994) e Almeida ${ }^{1}$ (1998). 


\section{RESULTADOS}

Os dados coletados foram inicialmente categorizados, de forma a permitir tratamento estatústico descritivo e inferencial-estatístico, em termos de características de perfil sociocultural e resultados do MEEM, bem como efeitos isolados e combinados de determinadas variáveis sobre o desempenho obtido no MEEM. A partir do referido tratamento, foram obtidos os resultados que são apresentados nas subseções seguintes. Dados acerca da caracterização sociodemográfica da amostra são resumidos pela tabela 1 .

Tabela 1 - Freqüência e percentual de ocorrência relativa aos dados sociodemográficos da amostra (n=65). Recife 2005

\begin{tabular}{|c|c|c|c|}
\hline \multicolumn{2}{|c|}{ Dados Sociodemográficos } & \multirow{2}{*}{$\begin{array}{l}\text { Freqüência } \\
12\end{array}$} & \multirow{2}{*}{$\begin{array}{l}\text { Percentual } \\
18,5\end{array}$} \\
\hline Idade em anos & 60 a 64 & & \\
\hline categorizada & 65 a 69 & 24 & 36,9 \\
\hline & 70 a 74 & 23 & 35,4 \\
\hline & 75 ou mais & 6 & 9,2 \\
\hline \multirow[t]{2}{*}{ Gênero } & Feminino & 62 & 95,4 \\
\hline & Masculino & 3 & 4,6 \\
\hline \multirow[t]{4}{*}{ Estado Civil } & Solteiro & 8 & 12,3 \\
\hline & Casado & 24 & 36,9 \\
\hline & Viúvo & 24 & 36,9 \\
\hline & Descasado & 9 & 13,8 \\
\hline \multirow{2}{*}{$\begin{array}{l}\text { Nível Sócio- } \\
\text { econômico }\end{array}$} & Não renda & 3 & 4,6 \\
\hline & 1 a 4 & 45 & 69,2 \\
\hline \multirow{4}{*}{$\begin{array}{l}\text { em número de } \\
\text { salários mínimos }\end{array}$} & 5 a 8 & 9 & 13,8 \\
\hline & 9 a 12 & 4 & 6,2 \\
\hline & 13 a 16 & 1 & 1,5 \\
\hline & 17 a 20 & 3 & 4,6 \\
\hline \multirow[t]{4}{*}{ Cor ou raça } & Branca & 35 & 53,8 \\
\hline & Parda & 24 & 36,9 \\
\hline & Preta & 5 & 7,7 \\
\hline & Indígena & 1 & 1,5 \\
\hline \multirow[t]{4}{*}{ Religião } & Católica & 53 & 81,5 \\
\hline & Espírita & 6 & 9,2 \\
\hline & Evangélica & 4 & 6,2 \\
\hline & Não tem & 2 & 3,1 \\
\hline \multirow[t]{5}{*}{ Escolaridade } & Analfabeto & 2 & 3,1 \\
\hline & Nível & 22 & 33,8 \\
\hline & Fundamental & 24 & 36,9 \\
\hline & Nível Médio & 17 & 26,1 \\
\hline & $\begin{array}{l}\text { Nível Superior e } \\
\text { pós-graduado }\end{array}$ & & \\
\hline
\end{tabular}


No que se refere às causas específicas de morbidade, 83,1\% dos idosos entrevistados mencionaram ter pelo menos uma doença ou condição crônica. Destes, 59,3\% relataram ter hipertensão, seguida de problemas de coluna e artrose (ambos com 18,5\%), osteoporose 14,8\%, colesterol - 13\%, doença do coração - 11,1\%, artrite - 9,3\% e diabetes - 3,7\%. Outras doenças foram lembradas, mas suas freqüências foram muito baixas, com um ou duas citações. Vale ressaltar que cada um dos participantes poderia indicar mais de uma doença ou condição crônica e que esse levantamento foi feito através de morbidade autoreferida, podendo o sujeito participante subestimar as prevalências de doenças ou condições crônicas, por problema de memória ou ausência de diagnóstico médico.

Há ainda um outro indicador da condição de saúde, que se refere ao fato de ter deixado de realizar atividades rotineiras por problema de saúde nas últimas duas semanas: $16,9 \%$ do total de participantes disseram ter passado por tal situação. Com relação à percepção acerca da própria saúde, os idosos entrevistados, em sua grande maioria, consideraram sua saúde como boa ou muito boa (93,8\% de respostas). No entanto, 66,2\% mencionaram alguma dificuldade de memória, sendo esta freqüente ou muito freqüente para $58,1 \%$ dos idosos com queixa e rara ou muito rara para 41,9\% deles. Além disso, $73,8 \%$ disseram praticar alguma atividade física. Destes, a atividade mais citada foi caminhada, com 44,6\%, seguida de hidroginástica, com 23,1\%, e ioga, com 13,8\% (observe-se, para a interpretação destes percentuais, que cada participante poderia mencionar mais de uma atividade física).

A análise descritiva do desempenho no MEEM dos participantes mostrou um escore mínimo de 22 pontos, um máximo de 30 e média de 26,9.

A análise de variância em que se verificou o efeito do nível de escolaridade e idade dos participantes sobre o desempenho no MEEM evidenciou efeito isolado significativo da escolaridade $\mathrm{F}=8,703$, p <0,000, 64g.l., mas nenhum efeito significativo isolado da idade $\mathrm{F}=0,442$, $\mathrm{p}=0,724$, 64g.l. sobre os escores do MEEM. A análise post-hoc para verificação de contrates (Tukey HSD Test) revelou diferenças significativas de escores MEEM entre analfabetos e sujeitos de nível médio e sujeitos de nível superior e pós-graduados, bem como diferença significativa entre os sujeitos com nível de escolaridade fundamental e sujeitos de nível médio e superior e pós-graduados, conforme resumido pela tabela 2 e pelo diagrama. 
Tabela 2 - Significância de contrastes de desempenho no MEEM em função de diferenças de níveis de escolaridade

\begin{tabular}{lllll}
\hline Escolaridade & Analfabetos & Fundamental & Médio & Superior e Pós \\
\hline Analfabetos & - & - & - & - \\
Fundamental & - & - & - & - \\
Médio & 0,004 & 0,038 & - & - \\
Superior e Pós & 0,001 & 0,003 & - & - \\
\hline
\end{tabular}

Diagrama - Significância de contrastes de desempenho no MEEM em função de diferenças de níveis de escolaridade (setas pontilhadas $=$ contraste estatisticamente significativo)
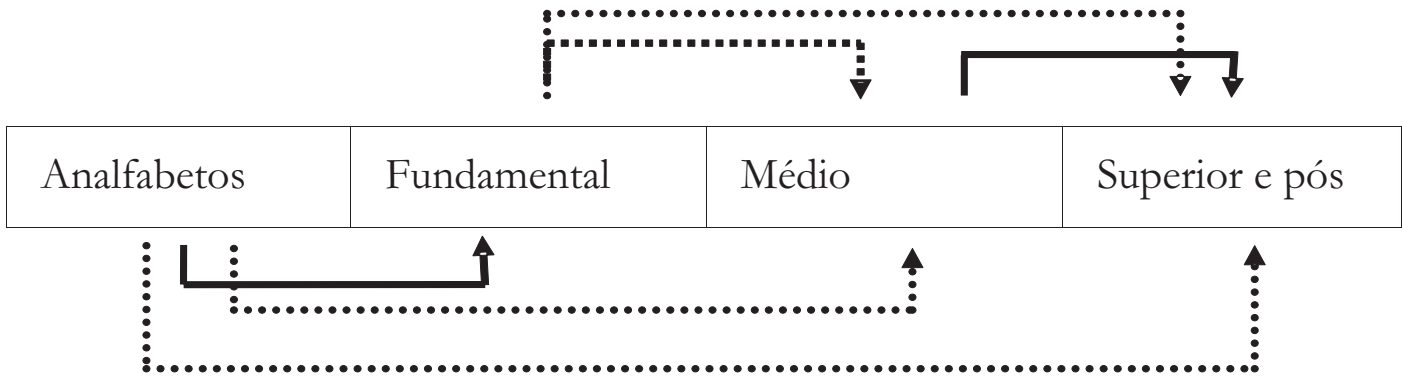

A média de escores do MEEM foi crescente, de acordo com o aumento da escolaridade. Ou seja, idosos analfabetos tiveram a média no MEEM de 22,5 pontos; os do nível fundamental, 25,9; o nível médio, 27,4; e o nível superior e pós-graduados, 28,1 (tabela 3).

Tabela 3 - Escores no MEEM e níveis de escolaridade

\begin{tabular}{llll}
\hline Escolaridade & $\begin{array}{l}\text { Média do } \\
\text { MEEM }\end{array}$ & $\begin{array}{l}\text { Escore Mínimo no } \\
\text { MEEM }\end{array}$ & $\begin{array}{l}\text { Escore Máximo } \\
\text { no MEEM }\end{array}$ \\
\hline Analfabetos & 22,5 & 22 & 23 \\
Nível Fundamental & 25,9 & 22 & 29 \\
Nível Médio & 27,4 & 23 & 30 \\
Nível Superior e pós- & 28,1 & 25 & 30 \\
graduados & & & \\
\hline
\end{tabular}


A análise da avaliação da satisfação global com a vida evidenciou que a maioria dos participantes $(67,7 \%)$ atribui escores entre 8 e 10 a esta questão (avaliações próximas do extremo "Melhor vida"), seguida dos escores 5 a 7, com 30,8; e por fim, 1 a 4 (avaliações próximas do extremo "Pior vida"), com 1,5\%.

\section{DISCUSSÃO E CONCLUSÕES}

O MEEM é um instrumento psicométrico usado mundialmente para avaliar estados demenciais em pessoas idosas, tanto normais como portadoras de quadros demenciais, como a doença de Alzheimer. O presente estudo teve como proposta usar o MEEM em idosos presumivelmente saudáveis, que estivessem vivendo em comunidade familiar e extrafamiliar. Os resultados encontrados confirmaram uma influência significativa da educação sobre os escores do MEEM - ou seja, quanto mais anos de estudo o sujeito tiver, maiores escores ele atinge quando submetido à avaliação através desse instrumento. Estudos de Bertolucci ${ }^{4}$ et al. (1994) e Almeida ${ }^{1}$ (1998) corroboram este dado. No entanto, os mesmos autores referem efeito significativo da idade sobre o MEEM, o que não foi verificado no presente estudo.

O efeito da variável independente "escolaridade" sobre o desempenho no teste MEEM, encontrado neste e em outros trabalhos, se coaduna com a estrutura desse instrumento: o MEEM é uma escala composta por itens que avaliam orientação para tempo e local, registro de palavras, atenção e cálculo, lembrança de palavras, linguagem e capaci- dade construtiva visual. Sendo assim, sobretudo o tópico de linguagem - para o qual se exigem leitura, escrita, atenção e cálculo, que requerem um desenvolvimento lógico-formal do indivíduo -, são itens difíceis de serem respondidos por pessoas de pouca ou nenhuma escolaridade.

Considerando a influência da educação sobre o MEEM, Bertolucci ${ }^{4}$ et al. (1994) propuseram que o ponto de corte para o diagnóstico genérico de "declínio cognitivo" em sujeitos brasileiros fosse de 13 pontos para analfabetos, 18 pontos para escolaridade bai$\mathrm{xa} /$ média e 26 pontos para alta escolaridade. Entretanto, segundo Almeida ${ }^{1}$ (1998), o corte para diagnóstico de demência em pessoas idosas sem escolaridade avaliadas pelo MEEM deveria ser de 19/20 pontos e para as escolarizadas, $23 / 24$ pontos. Em nosso estudo, observamos que os idosos entrevistados apresentaram escores acima dos propostos por Bertolucci e próximos aos sugeridos por Almeida. O grupo dos analfabetos entrevistados atingiu 22 pontos como menor escore; nos demais grupos de escolarização, o nível fundamental obteve 22 pontos; o médio, 23 pontos, e superior e pós-graduados, 25 pontos. Isso demonstra que os idosos participantes não se encontram em estados demenciais ou de declínio cognitivo, independentemente do nível de escolaridade, de acordo com os parâmetros do instrumento utilizado.

O perfil epidemiológico da amostra nos revela que, apesar dos critérios restritivos de composição da amostra, a grande maioria dos idosos entrevistados relatou ter pelo menos uma doença ou condição crônica, o que co- 
incide com os dados de Lima-Costa ${ }^{6}$ (2004), ao nos indicar que $69 \%$ dos idosos brasileiros também fazem tal relato. Um grande percentual desses idosos tem a hipertensão como doença mais freqüente, o que também vai ao encontro das informações da mesma autora. Além disso, os idosos entrevistados demonstraram bastante satisfação com seu estado de saúde, pois em sua grande maioria consideraram sua saúde como boa ou muito boa. Ressaltamos que esta variável, segundo Lima-Costa $^{6}$ (2004), em sua pesquisa, apresenta diferenças entre os idosos paulistas, com valor de $70 \%$, e os cariocas, com $44 \%$. Os dados aqui obtidos corroboram, os dados obtidos em São Paulo. Em relação ao indicador de saúde (não-realização de atividades rotineiras), observamos que o percentual de incidência desse indicador $(16,9 \%)$ está próximo dos índices nacionais, de 14\%.

No que se refere aos aspectos sociodemográficos, houve a esperada predominância percentual de presença de mulheres em relação aos homens na amostra aqui constituída. Além dos aspectos demográficos já aludidos, podemos pensar que os homens idosos estejam mais reclusos em suas casas, socialmente isolados, com menor disponibilidade para engajamento em projetos voltados para terceira idade, como aqueles promovidos pela UnATI, por exemplo, e/ ou disponíveis para participação em pesquisas acadêmicas.

Verificamos que os idosos participantes deste estudo se encontram inseridos nos dados epidemiológicos nacionais. Em relação aos dados do MEEM, os idosos apresentam-se saudáveis, não-enquadráveis em estados demenciais. Apesar de ainda persistirem atitudes de desvalorização social em relação a esse segmento etário, os dados sugerem que os idosos pesquisados estão incluídos numa situação de "velhice bem-sucedida", nos moldes propostos por $\mathrm{Neri}^{8}$ (2004), que descreve o idoso dessa categoria como sendo uma pessoa que mantém a autonomia, independência e envolvimento ativo com a vida, sendo funcionalmente capaz e produtivo. Nesse sentido, os dados aqui coletados confirmam a idéia segundo a qual o processo de envelhecimento não pode ser assimilado necessariamente ao adoecimento e à degeneração patológica. O envelhecimento não inviabiliza o funcionamento sócio-psicocultural do indivíduo, apenas o força a mudanças e adaptações, como de resto em qualquer outra passagem de etapas no percurso da vida.

Estudos adicionais poderiam abordar outros aspectos relevantes do envelhecimento, como por exemplo, o desenvolvimento da sabedoria ("wisdom"), na linha proposta por Paul Baltes ${ }^{3}$ e colaboradores. Em psicologia cognitiva, tais estudos poderiam evoluir na direção de fornecer subsídios de resposta à seguinte pergunta: "Será que ao envelhecer o ser bumano passa a funcionar cognitivamente de outro modo, a fim de compensar déficits somáticos e assim permanecer socialmente adaptado?".

Outro aspecto relevante a se considerar em futuros estudos diz respeito à ultrapassagem de indicações baseadas apenas em escores, que deveriam ser complementadas por avaliações clínico-qualitativas voltadas para a abordagem de estados afetivo-emocionais ligados à esfera do autoconceito, auto-estima e representa- 
ção social da velhice e do envelhecimento. Tal aspecto é crucial para a compreensão de um processo complexo, o envelhecimento, que, além de inegáveis bases orgânicas, se insere num contexto psico-sócio-histórico-cultural que não pode ser ignorado.

\section{NOTAS}

a Mestra em Psicologia Cognitiva pela Universidade Federal de Pernambuco

b Doutor em Psicologia pela Université de Paris. Professor e pesquisador do departamento de psicologia da Universidade Federal de Pernambuco

c Doutora em Odontologia Preventiva e Social pela Universidade de Pernambuco - Professora Adjunto II e coordenadora do Núcleo de Atenção ao Idoso (NAI) da Universidade Federal de Pernambuco.

d Doutora em Engenharia de Produção pela Universidade Federal do Rio de Janeiro. Professora adjunta da Universidade Federal de Pernambuco.

\section{REFERÊNCIAS}

1. Almeida O. Mini-exame do estado mental e o diagnóstico de demência no Brasil. Arquivos de Neuropsiquiatria 1998; 56: 605-12.

2. Antunes HKM, Santos RF, Mello MT. Avaliação cognitiva. In: Matsudo SMM. Avaliação do Idoso: física e funcional. Londrina: Midiograf; 2005.
3. Baltes PB. Envelhecimento cognitivo: potencialidades e limites. Gerontologia. 1994; 2(1): 23-44.

4. Bertolucci PHF, Brucki SMD, Campacci SR, Juliano Y. O mini-exame do estado mental em uma população geral: impacto da escolaridade. Arquivos de Neuropsiquiatria 1994; 52: 1-7.

5. Instituto Brasileiro de Geografia e Estatísticas. Pesquisa Nacional por Amostra de Domicílio (PNAD). Rio de Janeiro; 2004.

6. Lima-Costa MF. Epidemiologia do Envelhecimento no Brasil. In: Rouquayrol MZ, Almeida Filho N. Epidemiologia e saúde. Rio de Janeiro: MEDSI; 2004.

7. Miranda ML. Avaliação Psico-social. In: Matsudo SMM. Avaliação do idoso: física e funcional. Londrina: Midiograf; 2005.

8. Neri AL. Apresentação. In: Neri AL, Yassuda MS, orgsanizadores. Velhice bem-sucedida: aspectos afetivos e cognitivos. Campinas: Papirus; 2004.

9. Veras R. A novidade da agenda social contemporânea: a inclusão do cidadão de mais idade. A terceira idade 2003; 14: 6-29.

Recebido para publicação em 19/05/2006 Aceito em 28/11/2006 